\title{
Development of The System Detecting for Motor Abnormalities of Drone Using Temperature Sensor
}

\author{
Keita Kihara ${ }^{\mathrm{a},{ }^{*}}$, Huimin $\mathrm{Lu}^{\mathrm{b}}$, Shiyuan Yang ${ }^{\mathrm{a}}$, Seiichi Serikawa ${ }^{\mathrm{a}}$ \\ ${ }^{a}$ Department of Electrical and Electronics Engineering, Kyushu Institute of Technology, Japan \\ ${ }^{b}$ Department of Mechanical and Control Engineering, Kyushu Institute of Technology, Japan \\ *Corresponding Author: airgear7025@gmail.com
}

\begin{abstract}
Drone is widely used, such as weather observation, spraying of agricultural chemicals, inspection of infrastructure, monitoring of disaster sites where people and vehicles cannot enter. However, there are already 418 accidents of usage of drone in the US. In addition, there are solutions to other factors among the causes cited as factors of the fall accident, but there is no solution to the failure of the motor. The purpose of this paper is to develop a system which prevents the motor from being used under abnormal temperature conditions and in order to protect the drones without failing down. The abnormal temperature of drones' motors is monitored in this the proposed system. As a solution, Raspberrypi is attached to the drone to judge whether it was abnormal, and a temperature sensor was attached to the motors. Temperature sensor DS-18-B-20 is used for temperature measurement. We design an operation system to open the screen of Raspberrypi on PC for observation. In the proposed method, it is possible to stop the operation state of drone when abnormality exceeds the threshold setting of motor temperature. The experimental results demonstrate that the proposed system can realize more safe drones' unmanned flight by controlling drone from information obtained by attaching sensor to motor of drone
\end{abstract}

Keywords: Drone, Motor, Temperature sensor.

\section{Introduction}

Unmanned aerial vehicles traveling in the air by unattended and remote control are called drone, and are used in a wide range such as weather observation, spraying of agricultural chemicals, inspection of infrastructure, monitoring of disaster sites where people and vehicles cannot enter. Drone is strongly required to operate stably from disaster support and rescue, and also in the future that it can be used for carrying objects. However, 418 accidents have already happened in America.

One of the accidents of Drone is a fall accident, which includes short flight time due to inability to mount a large capacity battery, malfunction of motor and amp, communication loss etc. In order to reduce accidents, it is considered necessary to take measures against these factors. In this paper, researches on other factors are under way, whereas researches on breakdown of motors which are not being studied. As a solution to the failure of the motor, pay attention to the temperature which is a cause of failure of the motor, and construct a system for landing the drone when it reaches the temperature defined as abnormality.

We propose a falling countermeasure system using a temperature sensor and Raspberrypi for the purpose of preventing use under abnormal temperature of motor. A temperature sensor is attached to the motor part of the drone, and when it is judged that it is abnormal temperature on the Raspberrypi installed in the drone, it is automatically landing, and when it gets normal it is taken off again. There is no countermeasure system concerning drone's motor abnormality detection yet, it is considered to be a very meaningful research. With this countermeasure system, there is no fear of falling down the use of drone in environments where failures are not permitted, such as use for photographing purposes in the event of a disaster or investigation into areas where people do not enter, making it more secure and safer It is possible to do.

In addition, this automatic motor abnormality detection system increases safety, leading to more active use of automatic piloting, and consequently decreasing fall caused by communication loss caused by controller steering can be considered.

\section{Previous research}


There are various methods for detecting abnormality of the motor. For explaining why we adopted a method using a temperature sensor in this study, we give several methods of anomaly detection as an example. Why, in order to explain whether this technique adopted a method using a temperature sensor

First, there is an inspection system by abnormal sound detection. It is a method to automate an abnormal sound inspection using case-based identification algorithm. In this method, it is necessary to calculate the voice data by feature vector beforehand and calculate it based on the establishment distribution model, which is troublesome. In addition, considering that it is not always abnormal when it is abnormal, it is more meaningful for the system of this research that uses a temperature sensor that can reliably cope with a threshold value set as a motor abnormality. We selected a method using a temperature sensor.

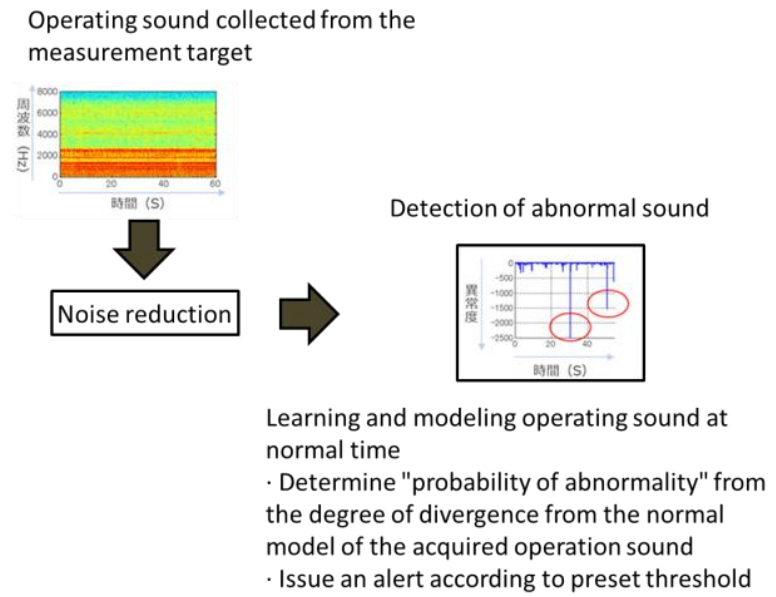

Fig. 1. Inspection system by abnormal sound detection

Next, there is a system in which a motor is photographed and an alarm is output when the detected temperature becomes equal to or higher than the set temperature in the thermal image. This has a problem that it is difficult to mount a camera on a drone having a limited loading amount, and it is costly to install a camera capable of taking a small thermal image. Also, it is difficult for the thermal image to judge the temperature change.

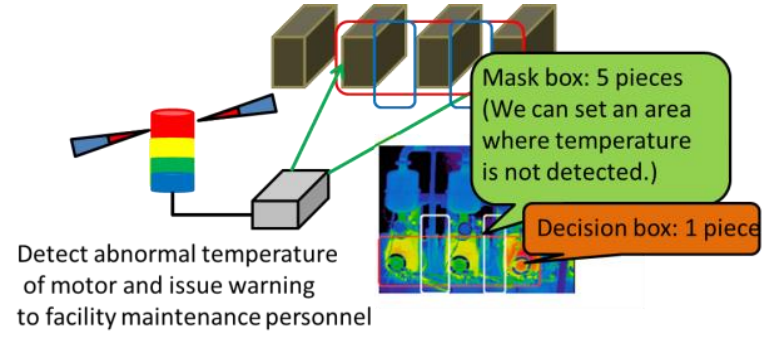

Fig. 2. A system in which a motor is photographed and an alarm

The fan sensor constantly monitors the rotation speed of the fan motor and outputs an alarm signal when abnormal rotation occurs. It is thought that it is difficult to judge whether the reduction of the rotation speed is due to abnormality or low speed in the drone that changes speed.

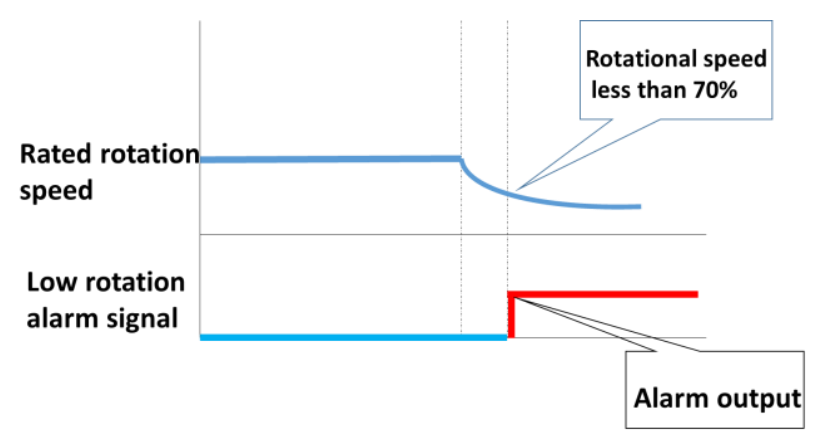

Fig. 3. Outline drawing of fan sensor system

As described above, the conventional abnormality detection method has a disadvantage in detecting abnormality of the droning motor. In this research, it was made possible to implement an anomaly detection system on a drone which was difficult with these methods using a temperature sensor (ds-18-b-20), Raspberrypi.

\section{The Proposed System}

In this paper, as a system to prevent use under abnormal temperature condition for the motor, we build a system to landing when the motor temperature exceeds a certain value.

If the temperature of the motor exceeds a certain threshold value as an abnormal condition, it is defined as abnormal. This is defined as it is said that degaussing will take place if the brushless motor mounted on the drone exceeds 70 to 80 degrees. In addition, in this study, since the drone's use time is as short as 10 minutes, $28^{\circ} \mathrm{C}$ is adopted as a substitute threshold for abnormality 
determination of $80^{\circ} \mathrm{C}$, based on the fact that the battery runs out before the temperature of the motor reaches $80^{\circ} \mathrm{C}$.

\subsection{System Overview}

Figure 4 shows the flow of the system flow in this study. The model proposed in this research consists of two devices, a drone real machine equipped with what is necessary for experiments and a hardware for observation. The drone side is equipped with a temperature sensor, Raspberrypi. Hardware for observation use laptop. A block diagram of the system is shown in Figure 5

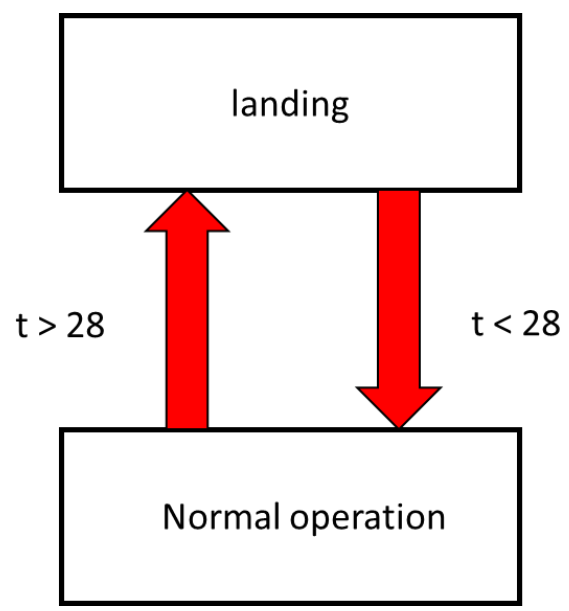

Fig. 4. System flow

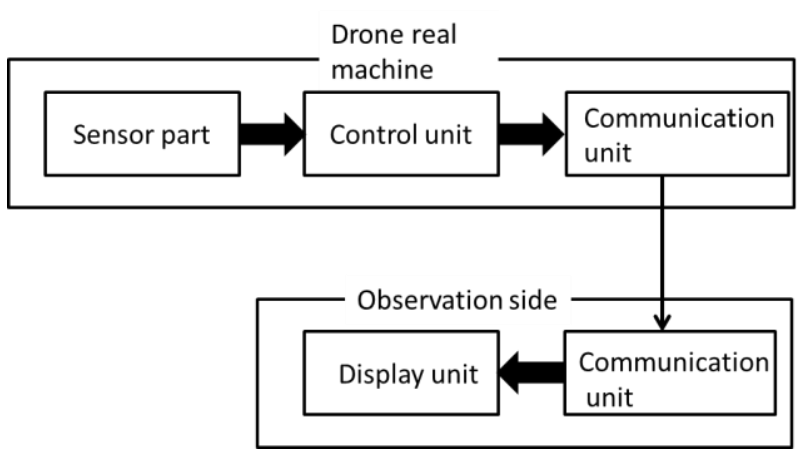

Fig. 5. System block diagram

\subsection{Sensor Setting}

\subsubsection{Drone Composition}

The structure of the drone is made up of four parts: a detection part, an actual machine (drone), a control part, and a communication part. Control and communication are done by one hardware. A temperature sensor was used for the detection part and Raspberrypi was used for the control part. As a control method, we implement our own python program on Raspberrypi and operate the drone's movement automatically. The communication section implements VNC and enables communication between Raspberrypi and the notebook PC. The configuration is shown in Figure 6

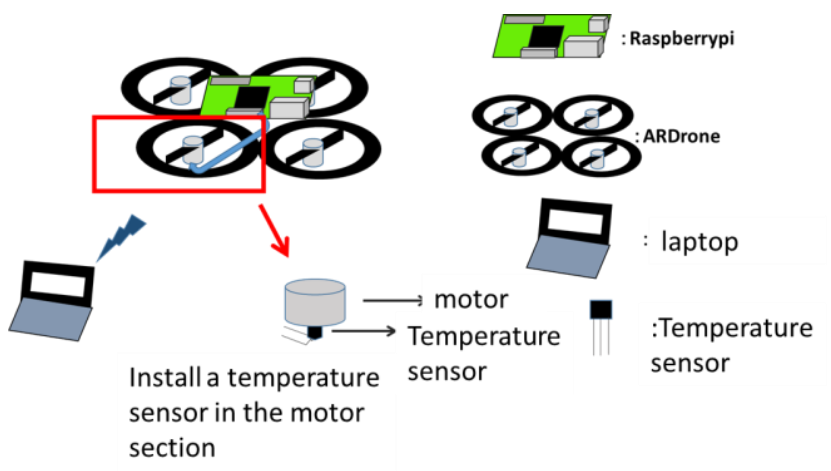

Fig. 6. Conceptual diagram of system

\subsubsection{Detection Unit}

A temperature measurement circuit using a temperature sensor is used for the detection section. The measurement circuit is shown in the figure.

In this study, a temperature sensor was installed as shown in the figure on the part where the motor was exposed (lower side of the motor case) to measure the temperature of the motor.

Because there are four motors of drone, it may be possible to install a temperature sensor on all four motors, but the disadvantage of increased weight due to installation and the fact that the four motors are flying while receiving the same load, We decided to install it on one motor because it can judge the state of other motors by measuring one motor.

The sensor used in this study is a 1-wire temperature sensor ds18b20. As 1-wire has an A/D converter built in the sensor itself, there is no signal deterioration due to wiring length and temperature data is converted to digital inside the sensor, so there is no error in signal transmission, and in RaspbianOS This sensor is used this time from the point that it can correspond to a 1-wire device by just loading a module for 1-wire. 


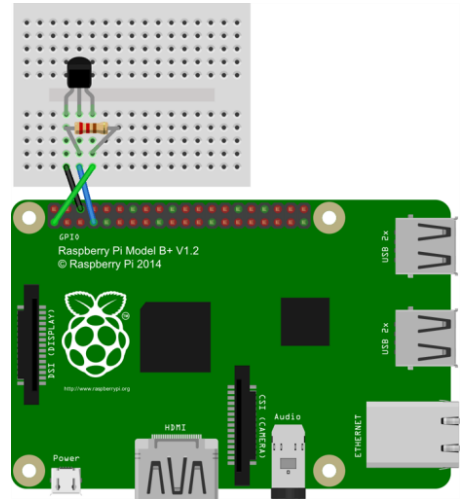

Fig. 7. Circuit diagram around the sensor

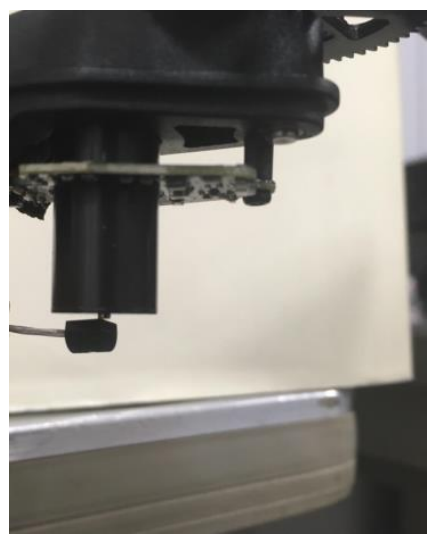

Fig. 8. Actual machine drawing (motor part)

\subsubsection{Control Unit}

The control unit uses Raspberrypi. Raspberrypi used this time is modelb + . This was developed as a single-board computer for education which is cheaper than the Raspberrypi Foundation. It has the feature that it can be used by installing power supply and SD card storage instead of mounting the internal hard disk etc. This time, modelB + has selected a function from a cheap price with a single function attached.

\section{Simulation Result}

In this paper, a system was constructed to achieve the purpose of landing when the motor temperature exceeded a certain value. Therefore, in the simulation, we confirmed whether this objective was achieved.

When the system was operated with the threshold set at $28^{\circ} \mathrm{C}$. It was confirmed that the system on Raspberrypi was judged to be abnormal and the drone was lowered when the motor temperature reached $28^{\circ} \mathrm{C}$ after the start of flight.

\section{Conclusions}

In this paper, we propose a motor abnormality detection system of an unmanned aircraft using a temperature sensor. We were able to construct a system to deal with motor abnormality, which is one of the causes of drone falling, using a temperature sensor and Raspberrypi. Even in the simulation, when it was measured that it exceeded 28 degrees, the landing was surely executed. By realizing this system, it is possible to deal with the troubles of the motor, which is supposed to occur when the drones are able to fly for a long time in the future.

\section{Acknowledgment}

This work was supported by JSPS KAKENHI (No.15F15077), Leading Initiative for Excellent Young Researcher (LEADER) of Ministry of Education, Culture, Sports, Science and Technology-Japan (16809746), Research Fund of Chinese Academy of Sciences (No.MGE2015KG02), Research Fund of State Key Laboratory of Marine Geology in Tongji University (MGK1608), and Research Fund of State Key Laboratory of Ocean Engineering in Shanghai Jiaotong University $(1315 ; 1510)$.

\section{References}

(1) NTT DATA "Started providing IOT solution to detect abnormality from working sound" NTT DATA,2016/10 /3,https://latte.la/column/43321221,(Reference2017-18)

(2) Nippon Avionics Co., Ltd," K-1. Motor abnormal temperature detection system | Infrared thermography | Nippon Avionics" Nippon Avionics Co., Ltd,1999, http://www.avio.co.jp/products/infrared/solution/k-hea vy/k-01.html,(Reference2017-1-8)

(3) Hirozawa Seiki Seisakusho Co., Ltd," Metal Feather Fan Sensor" Hirozawa Seiki Seisakusho Co., Ltd, http://www.hirosawaseiki.co.jp/ikurafan/pages/Cat/Ikur aFan1406_084.pdf,(Reference2017-1-8)

(4) Oriental Motor Co., Ltd," Design life of motor | Technical data |". Oriental Motor Co., Ltd, https://www.orientalmotor.co.jp/tech/reference/life01/,( Reference2017-1-8)

(5) TamuraYuuto,"Detection and deterioration diagnosis of electrical equipment based on current waveform pattern analysis" 
,2013/11/29,http://vision.kyoto-u.ac.jp/japanese/happy ou/pdf/Y-Tamura_ASN_20131129.pdf,(Reference2017 $-1-8)$ 